\title{
Dynamic Landscape of Mitochondrial Cytidine-To- Uridine RNA Editing in Tobacco (Nicotiana Tabacum) Shows its Tissue Specificity
}

\author{
Jing Fang \\ Wuhan Botanical Garden \\ Xiaohan Jiang \\ Wuhan Botanical Garden \\ Tengfei Wang \\ Wuhan Botanical Garden

\section{Zhiyu Deng} \\ Wuhan Botanical Garden
}

Aidi Zhang

Wuhan Botanical Garden

Xiujun Zhang ( $\square$ zhangxj@wbgcas.cn )

Wuhan Botanical Garden, Chinese Academy of Sciences https://orcid.org/0000-0001-8041-0592

\section{Research Article}

Keywords: RNA editing, Mitochondria, PPR gene, tissue specificity

Posted Date: July 7th, 2021

DOI: https://doi.org/10.21203/rs.3.rs-653672/v1

License: (9) (i) This work is licensed under a Creative Commons Attribution 4.0 International License. Read Full License

Version of Record: A version of this preprint was published at Plant Cell, Tissue and Organ Culture (PCTOC) on November 6th, 2021. See the published version at https://doi.org/10.1007/s11240-02102194-0. 


\section{Abstract}

The Cytidine-to-Uridine (C-to-U) RNA editing is a prevalent nucleoside modification at RNA level in plants. However, it is unclear whether the dynamics of C-to-U RNA editing is related to its tissues. In this study, we explored the tissue specificity of mitochondrial RNA editing based on RNA-seq data from tobacco (Nicotiana tabacum) root, stem, leaf, and flower tissues. As a result, a total of 331 RNA editing sites involving in 54 mitochondrial genes were identified. Among these identified RNA editing sites, 78 sites were confirmed tissues-specific. The results revealed dynamic landscape of conserved editing sites in editing efficiency among different tissues. To investigate the mechanism of tissue specificity of mitochondrial RNA editing in Nicotiana tabacum, the expression of RNA editing factor PPR genes was analyzed. The result shows that the expression level of $P P R$ genes in each tissue also varies from different tissues, indicating the heterogeneity of RNA editing in different tissues might result from the tissue specificity of $P P R$ genes expression. Our analyses provide insights into understanding landscape, regulation and function of RNA editing events in plants.

\section{Key Message}

Dynamic landscape of conserved editing sites revealed the tissue specificity of mitochondrial RNA editing in tobacco.

\section{Introduction}

RNA editing is defined as the insertion, deletion, and replacement of nucleotide bases that occurs after transcription, which usually results in difference between RNA genetic information and the genome template (Liscovitch-Brauer et al., 2017; Walkley and Li, 2017; Zahn, 2017; Peng et al., 2018). For instance, the transcript of maize chloroplast gene 50 S ribosomal protein $L 2(r p / 2)$ can only create the initiation codons after uridine replacement of cytidine (Hoch et al., 1991). In 1986, Dutch etbiologist Benne et al. discovered an RNA editing event in the mitochondrial gene cytochrome c oxidase subunit II (cox2) of trypanosomes firstly (Benne et al., 1986), they found that the insertion of four uridines caused the cox 2 gene to form a continuous open reading frame, resulting in changes in genetic information. In 1989, Hiesel et al. revealed that RNA editing also exists in primrose mitochondria (Hiesel et al., 1989). Subsequently, more and more RNA editing events were confirmed. To date, RNA editing has been found in primitive eukaryotes, vertebrates, plants, fungi and viruses (Palladino et al., 2000; Bahn et al., 2012; Alon et al., 2015; Guo et al., 2015; Riemondy et al., 2018; Zaidan et al., 2018).

In the case of higher plants, RNA editing mainly appears in the coding regions of mitochondrial and chloroplast genes, of which the most common editing style is cytidines (C) substituting uridines (U) (Covello and Gray, 1989; Tillich et al., 2006; Takenaka et al., 2013). So far, in higher plants, the total number of RNA editing sites in chloroplast genome (20-60) is much less than that of mitochondrial genome (300-600). During the process of RNA editing in higher plants, the editing factor recognizes a 20 $\sim 25$ nucleotide sequence upstream of the editing site, and the editing factors required for different RNA 
editing sites may differ (Yagi et al., 2014; Yan et al., 2018). A large number of studies have shown that RNA editing alters the genetic information from the genome and enriches the expression products of genes; on the other hand, it provides new genetic structures and functions for the evolution of organisms (Small et al., 2020; Lukeš et al., 2021). For the mammals, the regulation of RNA editing event has been widely studied. For example, the RNA editing in mammals has been proved to be dynamic landscape between tissues and more potential factors of editing have been discovered to be involved in the editing events (Tan et al., 2017; Blanc et al., 2019).

RNA editing in plant is mainly mediated by editing complex involving editing factors such as Pentapeptide repeat protein (PPR) protein and MORF protein (Tian et al., 2019). The PPR proteins are the largest class of RNA editing factors in plants, which is encoded by nuclear genes and located in mitochondria or chloroplast, playing a role of site-specific recognition in editing (Yagi et al., 2013; Yagi et al., 2014; Ichinose and Sugita, 2017). PPR proteins have two subfamilies, P-type and PLS-type, the P-type consists of the 35-amino acid classic PPR (P) motif, while the PLS-type consists of the classic P-motif and its variants $L$ (35 or 36 amino acids) and S (31 amino acids) (Manna, 2015). In fact, a plant-specific conserved E domain often exists at the C-terminus of the PLS type. Generally in plant organelles, it is the PLS-type PPR protein that recognizes the specific editing sites (Shikanai, 2015; Yan et al., 2018). However, the target relationship of $P P R$ protein catalyzing RNA editing site is still unclear.

Previous studies have revealed that RNA editing is specific in terms of tissues and developmental stages, suggesting that RNA editing may function as a regulatory device in plastid gene expression (Bock et al., 1993; Zeltz et al., 1993; Miyata and Sugita, 2004). Taking spinach as an example, reduced editing was observed in the photosystem II reaction center subunit VI (psbF) and PSII reaction center subunit XII $(p s b L)$ transcript from seeds and roots. Tissue-specific and development-specific RNA editing were also detected in the bryophyte ribosomal protein S14 (rps14) gene in previous studies (Miyata and Sugita, 2004). Tseng also observed that the RNA editing efficiency of Arabidopsis plastid mRNA was variable among different tissues (Tseng et al., 2013). Actually, earlier researches on RNA editing mainly focused on selected genes based on experimental methods with disadvantage of low throughput. With the rapid improvement of genomic and transcriptome sequencing technology, more and more genomes of plants have been sequenced and a great quantity of RNA-seq data have also been generated, which offers an opportunity to test the function and regulatory mechanism of RNA editing in plant growth and development. In the future, the regulation and function of RNA editing in plants and their effects on traits, especially some essential agronomic traits, will attract more people's attention (Small et al., 2020).

As a model plant, tobacco (Nicotiana tabacum) plays a key role in plant molecular research, and is also an important worldwide economical plant. In this study, in order to illustrate the tissue distribution characteristics of RNA editing in plant, we identified and analyzed RNA editing sites in four tobacco tissues (roots, stems, leaves, and flowers) based on transcriptome data. And then, the dynamic landscape of C-to-U RNA editing sites was analyzed and we discovered that editing sites were distributed differently in the four tissues, among which the editing sites in the root were the least, which might be related to the physiological function requirements for the roots. Simultaneously, we also analyzed the expression levels 
of RNA editing factors PPR genes in the four tissues, and its heterogeneity of expression levels might partly explain the varied RNA editing events happening in different tissues. Our findings show that RNA editing are differentially regulated in various types of tissues (non-green vs green), and may contribute to functional differentiation of tobacco tissues.

\section{Materials And Methods}

\section{Data collection}

The transcriptome data of root, stem, leaf, and flower tissues in tobacco (see supplementary materials section) were downloaded fromhttps://www.ncbi.nlm.nih.gov/bioproject/PRJNA208209, with the data files consisting of three replicates of each tissue (Table S1). In addition, tobacco mitochondrial genome sequence and its corresponding genome annotation file (tbl format) were downloaded from NCBI data repository (https://www.ncbi.nlm.nih.gov; accession numbers: NC_006581.1).

\section{Data pre-treatment}

In total, the identification process of RNA editing sites was split into two steps. Firstly, we aligned the transcriptome data against the tobacco mitochondrial genome as reference and called SNPs. Secondly, RNA editing sites were identified based on the called SNP-calling results. In order to increase sequencing depth, we merged the three duplicates from one tissue into one sample. Concretely, we firstly utilized the 'fastaqc' tool to evaluate the transcriptome data (Brown et al., 2017), and aligned each tissue transcriptome data against reference by 'HISAT2' software under default parameters (Kim et al., 2015). Afterwards, the alignment results were sorted, removed, indexed, and sorted by using 'samtool' tool (Li et al., 2009). Finally, the 'bcftools' tool was used to identify SNPs and generate VCF files (Danecek and McCarthy, 2017).

\section{Identification of RNA editing sites}

Based on the SNP-calling results (in 'VCF' format) and genome annotation files (in 'tbl' format), the RNA editing sites were identified under default parameter values by using the 'REDO' tool(Manna, 2015). 'REDO' is a comprehensive application tool for identifying RNA editing events in plant organelles based on variant call format files from RNA-sequencing data. The 'REDO' tool only works with three input files, i.e. the variant call format (VCF) files (records for all sites), the genome sequence file (FASTA format), and the gene annotation file (feature table file in 'tbl' format, https://www.ncbi.nlm.nih.gov/projects/Sequin/table.html). Afterwards, aiming to reduce the false positives, the raw variants were filtered by comprehensive rule dependent and statistical filters, such as (1) quality control filter, (2) depth filter (DP>4), (3) alt proportion filter (alt proportion < 0.1), (4) multiple alt filter, (5) distance filter, (6) spliced junction filter, (7) indel filter, likelihood ratio (LLR) test filter $(L L R<10)$, (8) Fisher's exact test filter ( $p$-value $<0.01)$, and (9) complicated filter model. Finally, the corresponding annotation information files for all identified RNA editing sites were generated.

\section{Characteristic statistics of identified RNA editing sites}


The For each tissue, the resulted editing sites were used for further characteristic statistics, including statistics of editing number, editing types, editing efficiency, codon positions, amino acid changes, and involved genes. For one site, the value of RNA editing efficiency was expressed as the proportion between edited transcripts and total transcripts. If the site was edited, the $\mathrm{C} / \mathrm{G}$ base (wild type) should be altered to the T/A base (edited type), since one editing site could be detected hundreds of times via sequencing, the number of wild type $(C / G)$ or edited type $(T / A)$ of bases could then be counted at this particular site, then the editing efficiency at one site could then be calculated by the formula: depth of edited bases ( $T$ and A)/total read depth of bases. Furthermore, we compared the RNA editing efficiency between each two tissues, and RNA editing sites with statistical significance ( $p$-value $<0.01)$ were identified. Aiming to decipher the tendency of RNA editing efficiency for each tissue, cluster analysis and 'heatmap' plotting were also performed based on matrix of RNA editing efficiency, which was normalized by subtracting the row-wise mean from the values in each row of data and multiplying all values in each row of data by standard deviation value. 'Pheatmap' function in R was used to plot the 'heatmap', 'dist' function was used to calculate the distance matrix of different samples with the default 'euclidean' method, and 'hclust' function was used to compute the hierarchical clustering. Additionally, we picked out genes with significantly changes in editing sites for further functional analysis.

\section{Expression analyses of PPR genes}

The Aimed to obtain all tobacco PPR genes, one PPR protein sequence (UniProtKB ID: Q9SAD9) of Arabidopsis thaliana was used as query to search against the Nicotiana tabacum protein databases using BLASTP. All positive hits were retrieved for gene function annotation to blast against the Swiss-Prot protein database. Furthermore, in order to measure and compare the levels of gene expression of $P P R$ genes, we also performed transcriptome analysis for the RNA-seq data used in our study. The protocol that described in previous study was used for transcriptome analysis (Pertea et al., 2016). Concretely, treated reads from each sample were mapped to the reference genome with 'HISAT2'; 'Stringtie' was used for transcript assembly; 'samtools' was used to index, merge, sort, format convert, 'mpileup', and remove duplications against the aligned data with default parameters; Gene expression levels were measured by TPM (Transcripts Per Kilobase of exon model per Million mapped reads), and expression values of $P P R$ proteins were also normalized by the method mentioned above, and a heatmap was plotted in all samples using 'pheatmap' function in R.

\section{Results}

\section{Alignment of transcriptome data}

There were a total of 12 RNA-seq samples that extracted from four tobacco tissues (root, stem, leaf, and flower) in our study, each tissue had three replicates. We merged replicates of each tissue and aligned the transcriptome data against the tobacco mitochondrial genome as reference and then called SNPs. The tobacco mitochondrial genome used in our study is $430597 \mathrm{bp}$ in size and encodes 183 genes. To avoid data bias in sequencing depth that might affect the comparability among four tissues, we unified the 
number of reads in four tissues RNA-seq data, then a total of 93415792 reads were selected randomly for transcriptome alignment. At last, there were 18525, 55569, 53162 and 102269 reads in the root, stem, leaf, and flower tissues mapped onto the tobacco mitochondrial genome, with corresponding overall alignment rate was about $0.02 \%, 0.07 \%, 0.08 \%$ and $0.13 \%$ respectively. Hence, root has the lowest mapping rate, which might be due to the less expression of transcripts in root compared with other tissues.

\section{The overview of RNA editing in Nicotiana tabacum}

A total of 331 mitochondrial RNA editing sites were predicted in the four tobacco tissues, involving 54 genes. Specifically, in terms of tissue types, the number of RNA editing sites identified in different tissues (root, stem, leaf, and flower) corresponded to 132, 244, 209, and 258 respectively, involving 33, 43, 43 and 49 genes, with detailed information of RNA editing sites shown in Table 1 and Table S5.

The statistics of RNA editing types revealed that C-to-U is the most editing type, accounting for more than 92\% (Fig. 1A), in accordance with previous studies that other mismatches might be the result of sequencing errors and double transcription of nuclear genes and mitochondrial genes (Edera et al., 2018), with details in Table S2. There were 12 genes had more than 10 editing sites, NADH dehydrogenase subunit 5 (nad5) gene had the most editing sites, with 22 editing sites. The second-most gene, with 20 editing sites, was cytochrome $c$ maturation protein ( $c c m F M)$ gene. From the perspective of gene family, RNA editing events tended to occur in NADH dehydrogenase complex, suggesting RNA editing plays a key role in the correction of protein functions of the NADH dehydrogenase complex. Compared with previous findings of Edera (Edera et al., 2018), 134 novel editing sites were detected, and one third of them located in ribosomal protein transcripts.

\section{Characteristic statistics of C-to-U RNA editing site}

Out of the 305 identified editing sites, 88 (28.85\%), 192 (62.95ロ), and 25 (8.20ロ) were located in first, second, and third codon positions respectively. It means that RNA editing events occurred preferentially on the second codon and followed by the first codon (Fig. 1B). Consistent with existing conclusions, the statistics of editing type showed that there were far more editing events of non-synonymous mutations than synonymous mutations, with non-synonymous editing events up to $90 \%$, as shown in Table S3. In addition, the editing level of non-synonymous mutations was significantly higher than that of synonymous mutations (Fig. 1C), indicating that non-synonymous $\mathrm{C}$-to-U editing was favored by natural selection. We found that the amino acid changes tended to be hydrophobic, as shown in Supplementary Figure S1. Furthermore, the proportion of changes from hydrophilic to hydrophobic was the most, accounting for about $53 \%$, followed by changes from hydrophobic to hydrophobic. Overall, the most amino acid changes were Ser-to-Leu (25.9\%) and Pro-to-Leu (19\%), serine was hydrophilic, whereas Leucine and Proline were both hydrophobic. These changes of hydrophobicity could greatly restore the conservation of proteins and were essential for maintaining normal protein function (Fig. 1D). 
The above results were also in good agreement with each tissue's statistics, such as the amino acid changes, the proportion of hydrophobic amino acids in the root, stem, leaf, and flower issues were $39.02 \%, 36.12 \%, 39.69 \%$, and $40.50 \%$, and after editing, the proportions were increased to $84.55 \%$, $86.34 \%, 84.02 \%$, and $85.12 \%$ respectively (Supplementary Figure S1). As for RNA editing efficiency, the overall average value was up to 0.9 (Fig. 2), for each tissue, the RNA editing efficiency of root, stem, leaf, and flower correspond to $0.89,0.90,0.91$ and 0.90 respectively. Clustering and heatmap plotting were also performed based on normalized matrix of RNA editing efficiency, as is shown in Fig. 3. The result demonstrated uneven distribution and dynamic landscape among different tissues, i.e. root has not only the least number of editing sites but also the lowest editing efficiency.

\section{Tissue-specific analysis of tobacco RNA editing sites}

Based on all identified RNA editing sites in four tissues, we found that the distribution of editing sites was quite uneven among tissues, for example, RNA editing in cob_114 (genome position: 41934) occurred in the all the tissues except root; matR_326 (genome position: 5687) was only edited in leaf tissue, as shown in Fig. 4.

The venn diagram analysis (Fig. 5) illustrated that there were only 91 common editing sites shared in all four tissues, whereas numbers of tissue-specific editing sites in tobacco root, stem, leaf, and flower was $5,21,17$, and 35 accordingly (detailed information were listed in Table S4). Meanwhile, there were 5 tissue-specific editing genes. Two genes orf306 and orf 151 were edited specially in stem and leaf respectively, whereas three genes cytochrome $c$ maturation genes $(\mathrm{ccmB}, \mathrm{ccmFc}$ ) and 30 S ribosomal gene (rps14) were edited in flower specially. Only the orf151 gene had two RNA editing sites, whereas the rest four genes had merely one site respectively. And all the editing sites in these genes were located in the second position of codons. By comparing the distribution of RNA editing sites in various tissues (Supplementary Figure S2), we observed that the editing events of NADH dehydrogenase subunits transcripts was obviously reduced in root, which is in line with the results of previous study (ChateignerBoutin and Hanson, 2003). It is worth noting that cytochrome $c$ maturation genes had the most editing sites in flower, suggesting that more energy was required in mature flower to ensure normal physiological functions. Moreover, compared with root, transcripts of ribosomal genes and maturase-related genes owned more editing sites in stem, leaf, and flower, indicating that RNA-editing plays a key role in rebuilding functions of protein biosynthesis and RNA splicing for these tissues.

Although the overall average editing efficiency presented at a high level, certain sites still had varied editing efficiency among different tissues. For example, the editing efficiency of rps10_2 in root was 0.6, whereas it was nearly fully edited in the other tissues ( $>=0.95)$, as shown in Supplementary Figure 22 . Additionally, from the cluster relationship in Fig. 3, root node clustered farthest away from the other three tissues, followed by leaf node, whereas flower and stem nodes clustered closely and owned more editing sites, demonstrating the fact of heterogeneity of RNA editing pattern among different tissues. As the underground organ of fixation of plant to the soil and absorption of water, root does not perform 
photosynthesis and simultaneously lose the necessity of correction of normal functions of certain genes. Hence, RNA editing events in root was reduced obviously compared with other tissues.

\section{The expression pattern of PPR gene is different among tissues}

In order to investigate the reason for the varied RNA editing events among different tissues, we also evaluated the expression of RNA editing factors $P P R$ genes. After blast searching, a total of $321 P P R$ genes were identified in tobacco mitochondria, of which $317,317,316$, and $316 P P R$ genes were found in root, stem, leaf, and flower, respectively. Based on normalized matrix of $P P R$ genes expression, heatmap plotting was also performed, as is shown in Fig. 6 and Supplementary Figure S3, which demonstrated an uneven distribution among different tissues. The average expression level of $P P R$ genes in root was lower than that of other tissues ( $p$-value $<0.01$ ), and the expression level of the $P P R$ gene in the root showed a polarization distribution. Specifically, some of the genes were expressed at a low level, while others were at high level expression.

The distribution trends of $P P R$ genes expression in stem and flower were similar to each other, which was in accord with their cluster relationship based on RNA editing efficiency. On the whole, although $P P R$ genes were highly expressed in stems and flowers, they were not dominant in the number. Considering the huge number of editing sites, it was speculated that the proteins encoded by these $P P R$ genes might be involved in the recognition of multiple RNA editing sites. Another interesting phenomenon was that the number of $P P R$ genes that were highly expressed in more than one tissue was small. Correspondingly, if a $P P R$ gene was highly expressed in one tissue, it is at relatively low expression level of the gene in the other three tissues. Hence, all the above results suggest that these PPR genes may exhibit tissue specific expression, which may partly explain the reason for the varied RNA editing events among different tissues.

\section{Discussion}

As an important epigenetic mechanism that modified genome-encoded transcripts, RNA editing diversifies gnomically encoded information to expand the complexity of the transcriptome. In plants, RNA editing commonly occurs in plastid and mitochondria, and C-to-U is the core editing type. Previous studies revealed that RNA editing has various biological functions, including promoting RNA splicing by affecting the intron structures, and playing a central role in plant development and evolutionary adaptation (Ichinose and Sugita, 2017; Tang and Luo, 2018). Until now, discrepancy in RNA editing sites among tissues has only been detected in certain genes, such as $p s b F$ and $p s b L$ genes (Bock et al., 1993). It is still difficult to make general conclusions to what extent that discrepancy exists between them. Hence, in order to obtain an overview of the distribution of RNA editing in the model plant tobacco, we used the transcriptome data and preformed bioinformatics analysis to examine the editing profiles of RNA editing and expression of PPR genes in root, stem, leaf, and flower tissue. A total of 331 RNA editing sites involving in 54 mitochondrial genes were identified. Compared to other tissues, root has not only the least 
number of editing sites (132), but also the lowest average editing efficiency. Totally, we found 78 tissuespecific RNA editing sites and 5 tissue-specific edited genes in the four tissues. The expression of RNA editing factor $P P R$ genes also demonstrated a varied pattern among four tissues.

Our study revealed that RNA editing of tobacco mitochondria RNA editing was a dynamic process, some editing sites were fully edited, whereas the others were partially edited in other tissues. Most editing events might be functionally regulated by varying the extent of editing. If the proteins produced by edited and unedited transcripts had different functions, it would be beneficial for plants to have regulated RNA

editing. Hence, it was conceivable that dynamic RNA editing, not changes in the genomic DNA sequence, might provide a flexible way to increase plant fitness and adaptation to the constantly changing environment. Indeed, we observed the dynamic response of RNA editing to temperature in our previous study in grape (Zhang et al., 2019). We also found that quiet a lot editing sites that were subject to tissuespecific regulation were located in transcripts encoding subunits of NADH dehydrogenase. Compared with other tissues, the editing of NADH dehydrogenase subunits transcripts in root was significantly down regulated, which was consistent with the results of previous studies (Tseng et al., 2013). The stable and high editing efficiencies in non-root tissues ensured their correct assembly into functional complexes. In contrast, without RNA editing, the unedited transcripts in root might not be translated properly, suggesting that these transcripts were not essential for root function. Occasionally, transcripts that contained a partially edited site might generate different forms of a protein with distinct or overlapping functions and further increase the diversity of the encoded proteins, but the underlying mechanism of functional differentiation remains unclear.

The current hypothesis is that $P P R$ proteins recognize cis-elements around the editing sites, and then recruit the editing enzyme to specific transcripts to catalyze the C-to-U conversion (Schmitz-Linneweber and Small, 2008). Therefore, it is possible that RNA editing is regulated by the expression of editing factors and their interacting proteins, such as $P P R$ proteins. Hence, we identified $321 P P R$ genes distributed roughly the same in the four tissues, in spite of which, expression of $P P R$ genes demonstrated a varied pattern among four tissues. For root, the expression levels of $P P R$ genes were polarized in distribution. The $P P R$ genes in root presented a relatively low expression level, which partly explained the reduced RNA editing events happening in root. Further studies are needed to identify components of the editing machinery and their regulation relationships between $P P R$ genes and editing sites.

\section{Declarations}

Author Contributions: Conceptualization, J.F., X.J., A.Z. and X.Z.; methodology, A.Z. and J.F.; software, A.Z and J.F.; validation, J.F. and A.Z.; formal analysis, J.F. and A.Z.; investigation, J.F. and A.Z.; resources, J.F. and A.Z.; data curation, J.F., T.W., Z.D. and A.Z.\&writing-original draft preparation, J.F. and A.Z.; writingreview and editing, A.Z. and X.Z.; visualization, J.F.,X.J, and T.W.; supervision, X.Z.; project administration, X.Z.; funding acquisition, X.Z. All authors have read and agreed to the published version of the manuscript. 
Funding: This research was funded by the National Natural Science Foundation of China, grant numbers 32070682 and 31702322, the National Science \& Technology Innovation Zone Project, grant numbers 1716315XJ00200303 and 1816315XJ00100216, and CAS Pioneer Hundred Talents Program.

Acknowledgments: We would like to thank the members of Bioinformatics Group of Wuhan Botanical Garden, Chinese Academy of Sciences, China for the discussion and suggestion to improve the manuscript.

Conflicts of Interest: The funders had no role in the design of the study; in the collection, analyses, or interpretation of data; in the writing of the manuscript, or in the decision to publish the results.

\section{References}

1. Alon S, Garrett SC, Levanon EY, Olson S, Graveley BR, Rosenthal JJ, and Eisenberg E (2015) The majority of transcripts in the squid nervous system are extensively recoded by A-to-I RNA editing. Elife 4

2. Bahn JH, Lee JH, Li G, Greer C, Peng G, and Xiao X (2012) Accurate identification of A-to-I RNA editing in human by transcriptome sequencing. Genome Res 22:142-150

3. Benne R, Van den Burg, J., Brakenhoff, J.P., Sloof, P., Van Boom, J.H., and Tromp, M.C. (1986). Major transcript of the frameshifted coxll gene from trypanosome mitochondria contains four nucleotides that are not encoded in the DNA. Cell 46, 819-826

4. Blanc V, Xie Y, Kennedy S, Riordan JD, Rubin DC, Madison BB, Mills JC, Nadeau JH, and Davidson NO (2019) Apobec1 complementation factor (A1CF) and RBM47 interact in tissue-specific regulation of $\mathrm{C}$ to U RNA editing in mouse intestine and liver. RNA 25:70-81

5. Bock R, Hagemann R, Kossel H, and Kudla J (1993) Tissue- and stage-specific modulation of RNA editing of the $\mathrm{psbF}$ and $\mathrm{psbL}$ transcript from spinach plastids-a new regulatory mechanism? Mol Gen Genet 240:238-244

6. Brown J, Pirrung M, and McCue LA (2017) FQC Dashboard: integrates FastQC results into a webbased, interactive, and extensible FASTQ quality control tool. Bioinformatics 33:3137-3139

7. Chateigner-Boutin AL, and Hanson MR (2003) Developmental co-variation of RNA editing extent of plastid editing sites exhibiting similar cis-elements. Nucleic Acids Res 31:2586-2594

8. Covello PS, and Gray MW (1989) Rna Editing in Plant-Mitochondria. Nature 341:662-666

9. Danecek P, and McCarthy SA (2017) BCFtools/csq: haplotype-aware variant consequences. Bioinformatics 33:2037-2039

10. Edera AA, Gandini CL, and Sanchez-Puerta MV (2018) Towards a comprehensive picture of C-to-U RNA editing sites in angiosperm mitochondria. Plant Mol Biol 97:215-231

11. Guo WH, Grewe F, and Mower JP (2015) Variable Frequency of Plastid RNA Editing among Ferns and Repeated Loss of Uridine-to-Cytidine Editing from Vascular Plants. Plos One 10:e0117075 
12. Hiesel R, Wissinger B, Schuster W, and Brennicke A (1989) RNA editing in plant mitochondria. Science 246:1632-1634

13. Hoch B, Maier RM, Appel K, Igloi GL, and Kossel H (1991) Editing of a chloroplast mRNA by creation of an initiation codon. Nature 353:178-180

14. Ichinose M, and Sugita M (2017) RNA Editing and Its Molecular Mechanism in Plant Organelles. Genes 8:5

15. Kim D, Langmead B, and Salzberg SL (2015) HISAT: a fast spliced aligner with low memory requirements. Nat Methods 12:357-360

16. Li H, Handsaker B, Wysoker A, Fennell T, Ruan J, Homer N, Marth G, Abecasis G, Durbin R, and Genome Project Data Processing, S (2009) The Sequence Alignment/Map format and SAMtools. Bioinformatics 25, 2078-2079

17. Liscovitch-Brauer N, Alon S, Porath HT, Elstein B, Unger R, Ziv T, Admon A, Levanon EY, Rosenthal JJC, and Eisenberg E (2017) Trade-off between Transcriptome Plasticity and Genome Evolution in Cephalopods. Cell 169:191-202

18. Lukeš J, Kaur B, and Speijer D (2021) RNA Editing in Mitochondria and Plastids: Weird and Widespread. Trends Genet 37:99-102

19. Manna S (2015) An overview of pentatricopeptide repeat proteins and their applications. Biochimie 113:93-99

20. Miyata $Y$, and Sugita M (2004) Tissue- and stage-specific RNA editing of rps 14 transcripts in moss (Physcomitrella patens) chloroplasts. J Plant Physiol 161:113-115

21. Palladino MJ, Keegan LP, O'Connell MA, and Reenan RA (2000) A-to-I pre-mRNA editing in Drosophila is primarily involved in adult nervous system function and integrity. Cell 102:437-449

22. Peng $X, X u X$, Wang Y, Hawke DH, Yu S, Han L, Zhou Z, Mojumdar K, Jeong KJ, Labrie M, Tsang YH, Zhang M, Lu Y, Hwu P, Scott KL, Liang H, and Mills, G.B (2018) A-to-I RNA Editing Contributes to Proteomic Diversity in Cancer. Cancer Cell 33, 817-828

23. Pertea M, Kim D, Pertea GM, Leek JT, and Salzberg SL (2016) Transcript-level expression analysis of RNA-seq experiments with HISAT, StringTie and Ballgown. Nat Protoc 11:1650-1667

24. Riemondy KA, Gillen AE, White EA, Bogren LK, Hesselberth JR, and Martin SL (2018) Dynamic temperature-sensitive A-to-I RNA editing in the brain of a heterothermic mammal during hibernation. RNA 24:1481-1495

25. Schmitz-Linneweber C, and Small I (2008) Pentatricopeptide repeat proteins: a socket set for organelle gene expression. Trends Plant Sci 13:663-670

26. Shikanai T (2015) RNA editing in plants: Machinery and flexibility of site recognition. Biochimica Et Biophysica Acta-Bioenergetics 1847:779-785

27. Small ID, Schallenberg-Rüdinger $M$, Takenaka $M$, Mireau $H$, and Ostersetzer-Biran, $O$ (2020) Plant organellar RNA editing: what 30 years of research has revealed. Plant J 101, 1040-1056 
28. Takenaka M, Zehrmann A, Verbitskiy D, Hartel B, and Brennicke A (2013) RNA Editing in Plants and Its Evolution. Annu Rev Genet 47 47:335-352

29. Tan MH, Li Q, Shanmugam R, Piskol R, Kohler J, Young AN, Liu KI, Zhang R, Ramaswami G, Ariyoshi K, Gupte A, Keegan LP, George CX, Ramu A, Huang N, Pollina EA, Leeman DS, Rustighi A, Goh YPS, Chawla A, Del Sal G, Peltz G, Brunet A, Conrad DF, Samuel CE, O'Connell, M.A., Walkley, C.R., Nishikura, K., and Li, J.B. (2017). Dynamic landscape and regulation of RNA editing in mammals. Nature 550, 249-254

30. Tang W, and Luo C (2018) Molecular and Functional Diversity of RNA Editing in Plant Mitochondria. Mol Biotechnol 60:935-945

31. Tian F, Yu J, Zhang Y, Xie Y, Wu B, and Miao Y (2019) MORF9 Functions in Plastid RNA Editing with Tissue Specificity. Int J Mol Sci 20:4635

32. Tillich M, Lehwark P, Morton BR, and Maier UG (2006) The evolution of chloroplast RNA editing. Mol Biol Evol 23:1912-1921

33. Tseng CC, Lee CJ, Chung YT, Sung TY, and Hsieh MH (2013) Differential regulation of Arabidopsis plastid gene expression and RNA editing in non-photosynthetic tissues. Plant Mol Biol 82:375-392

34. Walkley CR, and Li JB (2017) Rewriting the transcriptome: adenosine-to-inosine RNA editing by ADARs. Genome Biol 18:205

35. Yagi Y, Nakamura T, and Small I (2014) The potential for manipulating RNA with pentatricopeptide repeat proteins. Plant J 78:772-782

36. Yagi Y, Tachikawa M, Noguchi H, Satoh S, Obokata J, and Nakamura T (2013) Pentatricopeptide repeat proteins involved in plant organellar RNA editing. RNA Biol 10:1419-1425

37. Yan J, Zhang Q, and Yin P (2018) RNA editing machinery in plant organelles. Sci China Life Sci 61:162-169

38. Zahn LM (2017) The evolution of edited RNA transcripts. Science 355:1278-1279

39. Zaidan H, Ramaswami G, Golumbic YN, Sher N, Malik A, Barak M, Galiani D, Dekel N, Li JB, and Gaisler-Salomon, I (2018) A-to-I RNA editing in the rat brain is age-dependent, region-specific and sensitive to environmental stress across generations. BMC Genomics 19, 28

40. Zeltz P, Hess WR, Neckermann K, Borner T, and Kossel H (1993) Editing of the chloroplast rpoB transcript is independent of chloroplast translation and shows different patterns in barley and maize. EMBO J 12:4291-4296

41. Zhang $A$, Jiang $X$, Zhang $F$, Wang $T$, and Zhang $X$ (2019) Dynamic response of RNA editing to temperature in grape by RNA deep sequencing. Funct Integr Genomics 20:421-432

\section{Tables}

Table 1. The statistics of identified RNA editing sites in four tissues of Nicotiana tabacum mitochondrion. 
Prefix Total Phase(1,2,3) Phase(2)/Totall Silent NonSilentt NonSilent/Total

\begin{tabular}{lllllll}
\hline root & 132 & $36,88,8$ & 0.6617 & 8 & 124 & 0.9323 \\
\hline stem & 244 & $73,147,24$ & 0.5977 & 25 & 219 & 0.8902 \\
\hline leaf & 209 & $58,130,21$ & 0.622 & 24 & 185 & 0.8852 \\
\hline flower & 258 & $76,159,23$ & 0.6163 & 27 & 231 & 0.8953
\end{tabular}

\section{Figures}
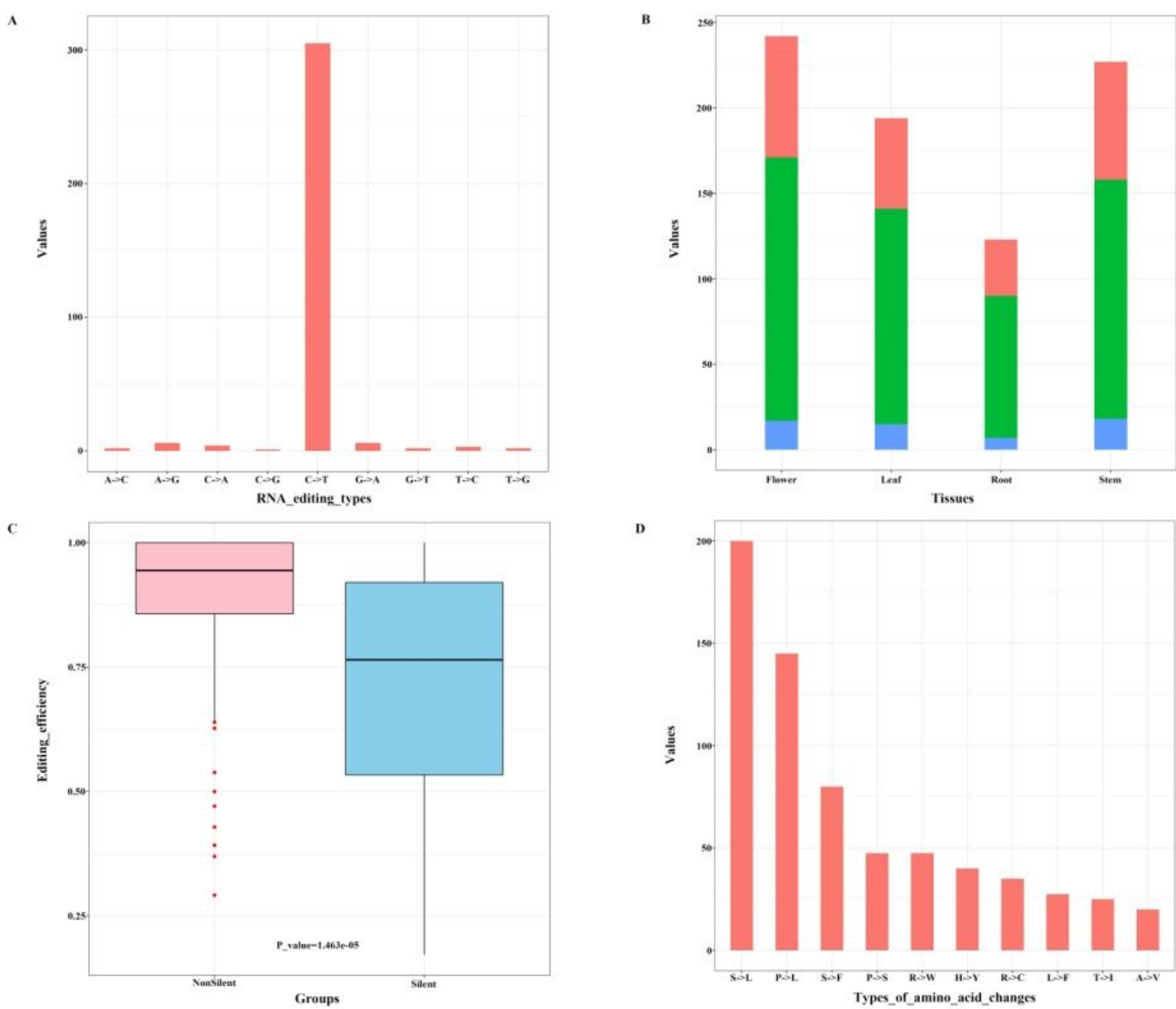

\section{Figure 1}

RNA editing in Nicotiana tabacum. (A) The editing types in Nicotiana tabacum. A total of 305 C-to-U RNA editing sites were identified, accounting for more than $92 \%$ of all sites. (B) Total number of C-to-U RNA editing sites in four tissues of Nicotiana tabacum. The stacked bars from top to bottom depict the number of editing sites at the first (red), second (green), and third (blue) codon positions, respectively. (C) C-to-U editing efficiency of non-synonymous (276) and synonymous (29) editing events. Discrete points are indicated in red. P-value is calculated by Wilcoxon rank sum test. Silent, synonymous editing; 
NonSilent, non-synonymous editing. (D) The changes of amino acid types in Nicotiana tabacum. 80 $(25.9 \%)$ out of 305 diversifications were Ser-to-Leu changes.

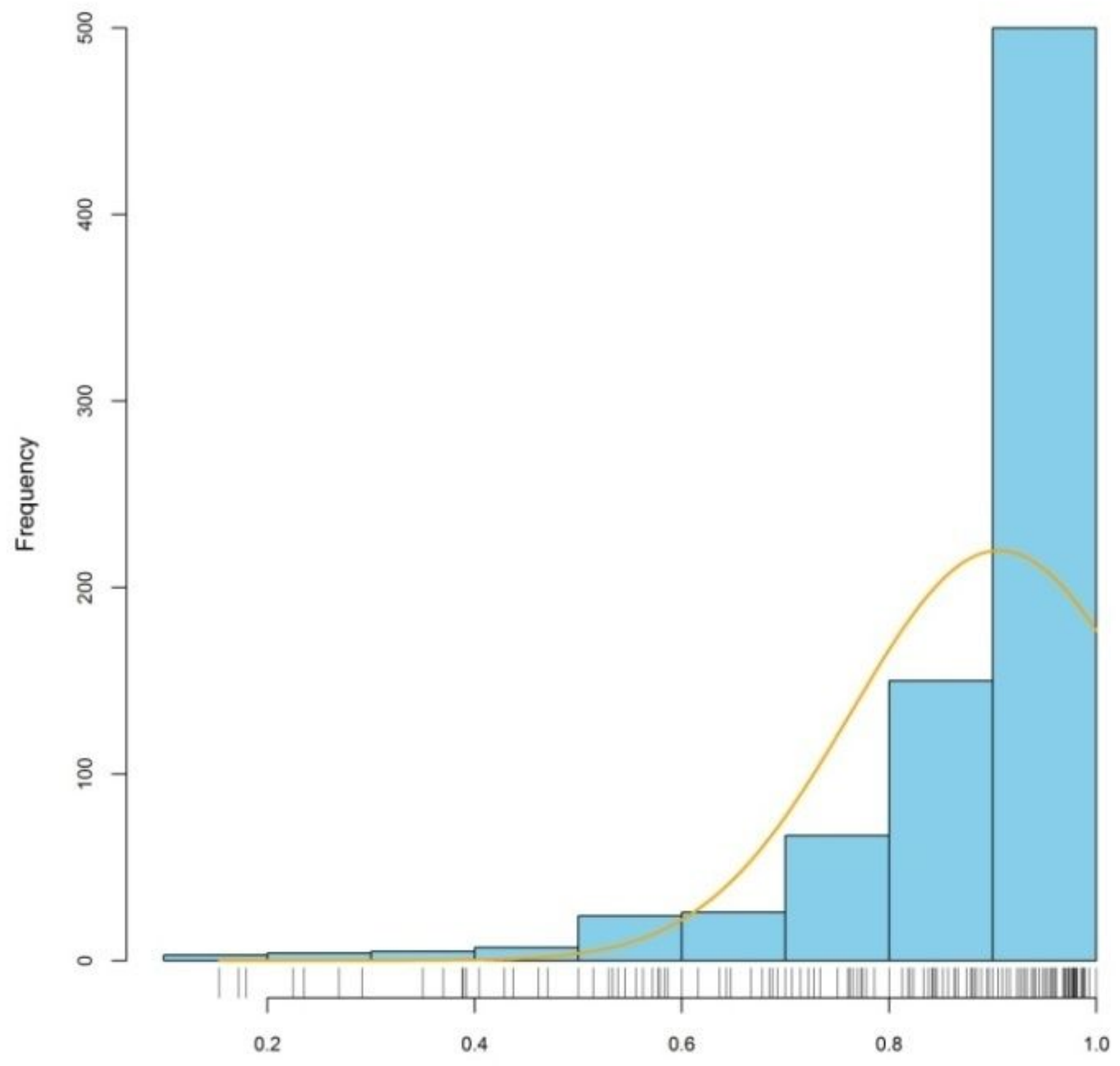

Editing_efficiency

\section{Figure 2}

RNA Distribution of editing efficiency in Nicotiana tabacum. The curve represents the normal density distribution of editing efficiency. 


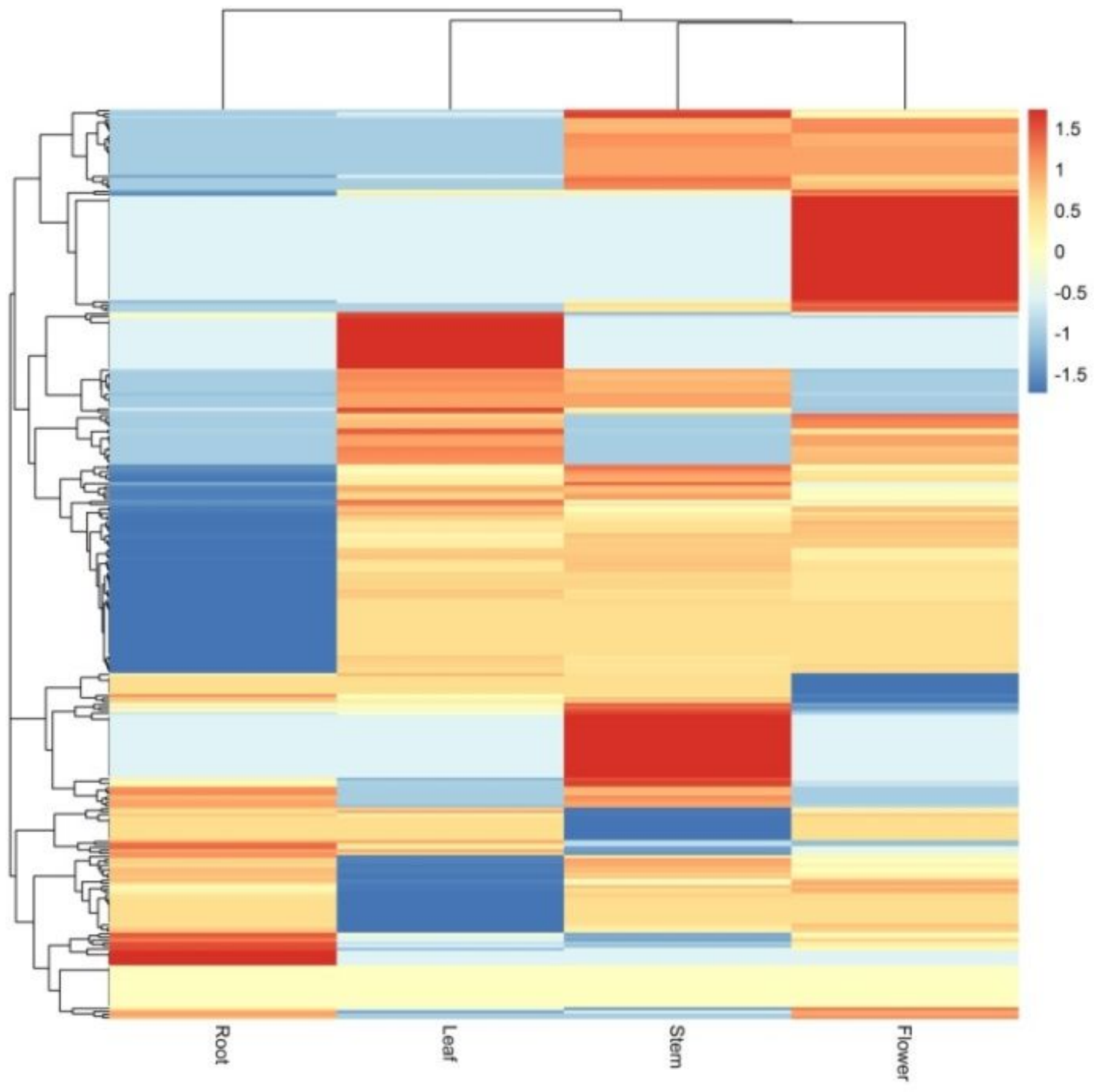

Figure 3

Heatmap of RNA editing efficiency in four tissues of Nicotiana tabacum. The $x$ axis represents different samples, and the y axis represents RNA editing sites. 


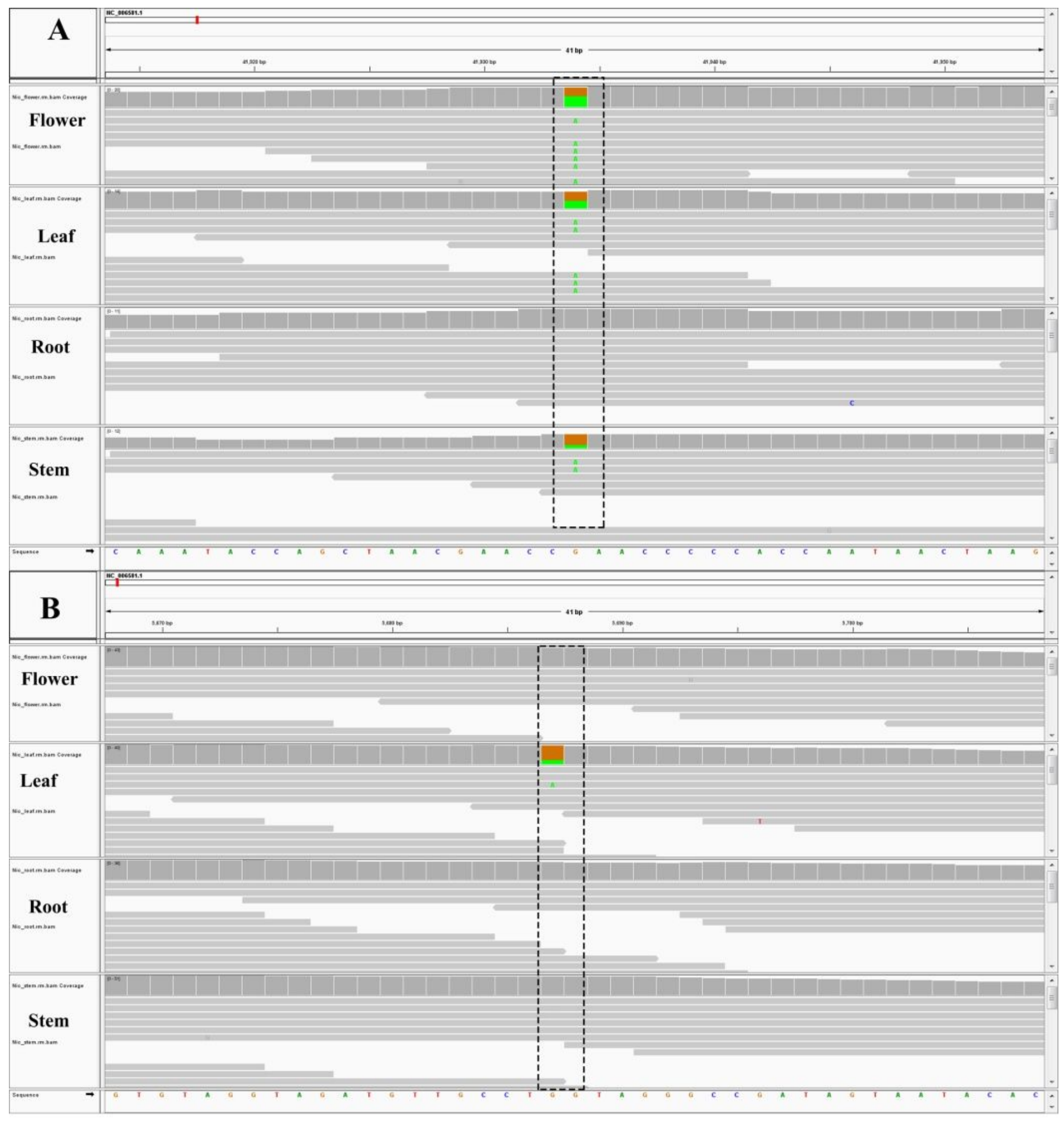

\section{Figure 4}

Snapshot of alignment of reads from four tissues against mitochondrial genome for two editing sites in Nicotiana tabacum. The emphasized sites were indicated by black dotted box. (A) Snapshot of editing site cob_114 (genome position: 41934) in four tissues of Nicotiana tabacum, demonstrating that this site was edited in all the four tissue except root. (B) Snapshot of editing site matR_326 (genome position: 5687) in four tissues of Nicotiana tabacum, demonstrating that this site was only edited in leaf tissue. 
A

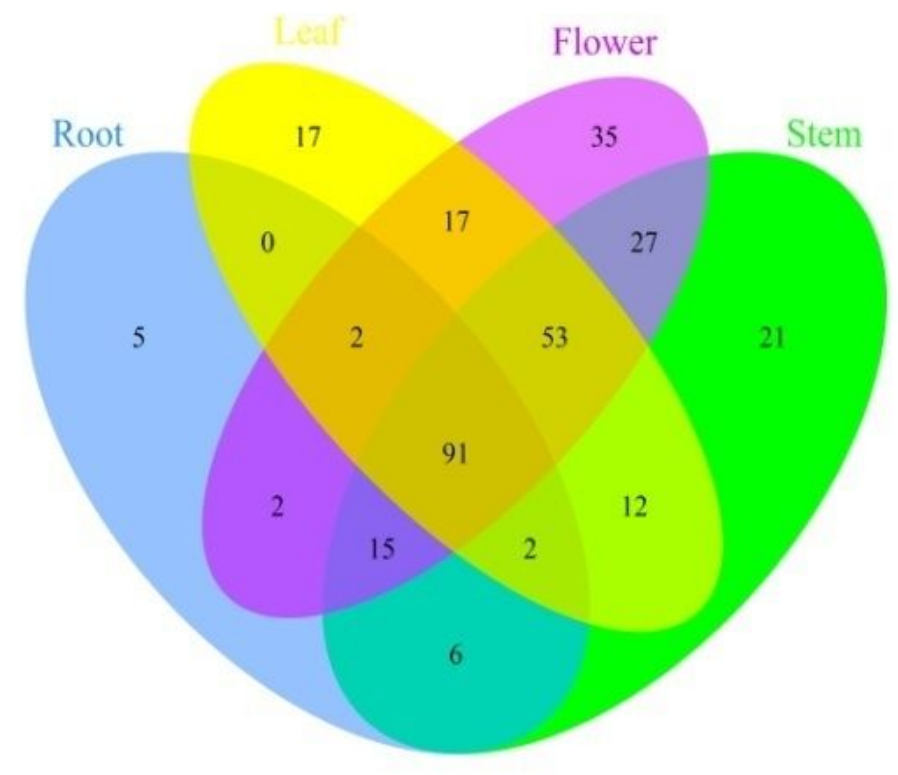

B

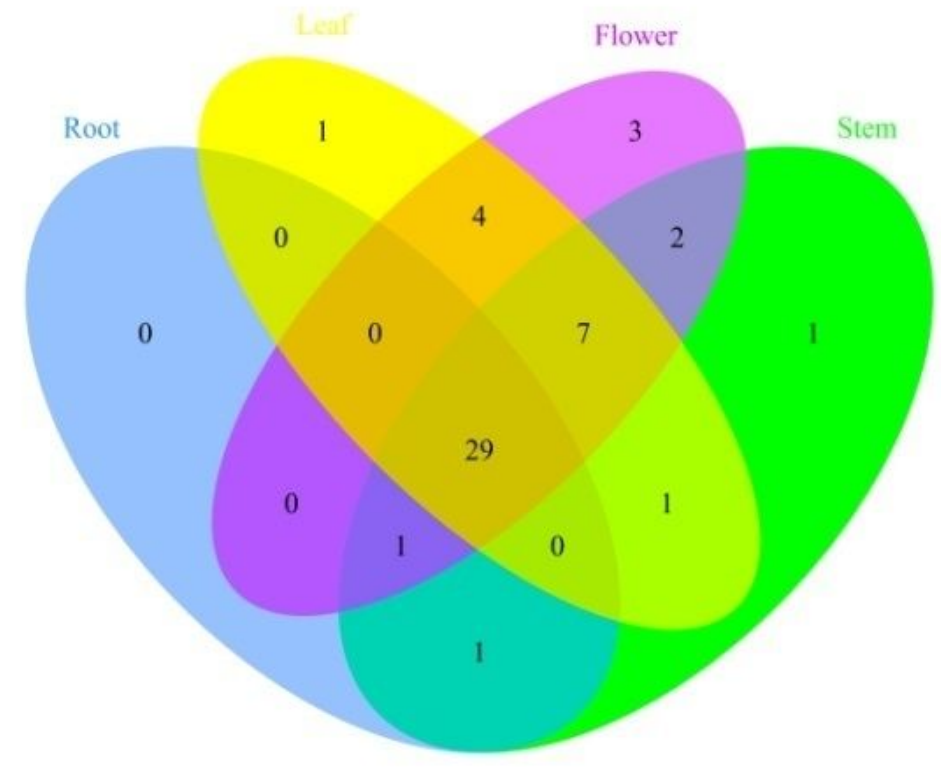

Figure 5

Venn diagram demonstration of tissue specificity of C-to-U RNA editing sites and genes. (A) Venn diagram demonstration of tissues specificity of C-to-U RNA editing sites. (B) Venn diagram demonstration of genes with tissues-specific RNA editing sites. 


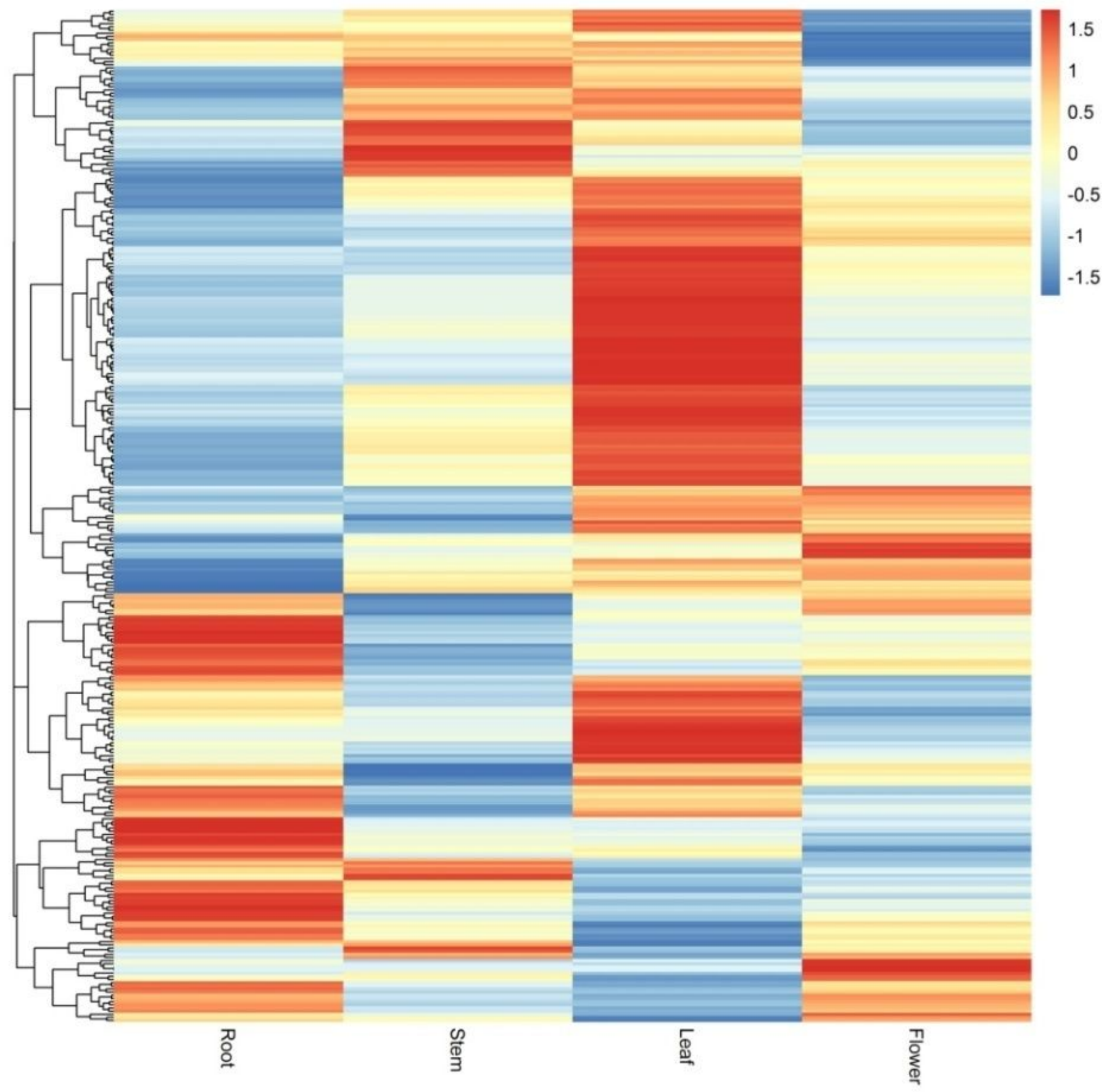

Figure 6

Heatmap of PPR protein expressions in four tissues of Nicotiana tabacum. The $\mathrm{x}$ axis represents different samples, and the $y$ axis represents PPR proteins.

\section{Supplementary Files}

This is a list of supplementary files associated with this preprint. Click to download. 
- SupplementaryFigures14andTables14.docx

- SupplementaryTables56.rar 